\title{
Atraumatic Extraction and immediate implant placement into infected site with the "ice cream cone" technique and L-PRF: A Case Report
}

\author{
Javier Basualdo ${ }^{1 *}$, Mariana Ivankovic ${ }^{1}$, Janja Kuzmicic ${ }^{2}$, Eduardo Fernández ${ }^{1}$
}

\author{
1. Facultad de Odontología, Universidad de Chile. \\ Chile \\ 2. Práctica Privada \\ * Corresponding Author: Javier Basualdo \\ Av. Santa María 0596, Providencia, Santiago, Chile \\ | Fono +56 989046734 | Email: drjavierbasualdo@ \\ gmail.com \\ Work received on 24/06/2017. Approved for \\ publication 29/10/2017
}

\begin{abstract}
We describe a case report of a 53 years old patient with osteopenia treatment, which presented a longitudinal root fracture in relation to 9 tooth Single Fixed Prothesis (SFP), an active fistula and a bucal plate loss. It was prescribed the tooth extraction and the immediate Biohorizons $₫$ Tapered Internal $®$ implant installation. The regeneration of the bucal plate was performed using the technique of "ice cream cone" using Mineross $₫$, Mem Lok@ and L-PRF.

KEYWORDS

Immediate implant, Guided bone regeneration (GBR), Infected site, Ice cream cone.
\end{abstract}

Rev. Clin. Periodoncia Implantol. Rehabil. Oral Vol. 11(1); 43-46, 2018.

\section{INTRODUCTION}

After a dental extraction, a reabsorption process occurs in the alveolar bone. Anatomically and histologically, the alveolar bone corresponds to a dependent structure of the dentition, which develops in conjunction with the eruption of the teeth. In the first phase of remodeling, the lingual plate and parts of the buccal plate of a post-extraction socket suffer bone loss due to the loss of the bundle bone, because its nutrition depends on the periodontal ligament. Therefore, there is a loss of bone, vertically and horizontally ${ }^{(1)}$,which can produce an aesthetic defect that is vitally important in the anterior sector. This aesthetic defect may worsen if the extraction technique is not correct, so it is recommended that an atraumatic technique be performed using traditional methods, specialized extractors, periotomes and endodontic files for the removal of radicular remains, or the use of piezo surgery ${ }^{(2)}$.

The immediate implant placement technique is currently a routine procedure with success rates like those for the installation of a conventional implant ${ }^{(3)}$. The immediate placement of an implant in a fresh socket in the aesthetic area is a complex process and involves great dexterity. It presents advantages such as: shorter treatment time, better aesthetic results and better patient comfort during the osseointegration period ${ }^{(4)}$. These reasons have led to the massification of this technique; it has been demonstrated that the immediate placement of implants in an infected site has success rates like those for immediate conventional placement, but more long-term studies are needed $(5,6)$.

The technique of bone regeneration known as "ice cream cone" is a technique that uses a collagen membrane in the form of an ice cream cone and bone filler material to regenerate the buccal plate of a fresh socket ${ }^{(7)}$. Elian et al. ${ }^{(8)}$ developed a post-extraction fresh socket classification system:

Type 1: The facial soft tissue and buccal plate of bone are at normal levels in relation to the cement enamel junction of the pre-extracted tooth and remain intact post extraction.

Type 2: Facial soft tissue is present but the buccal plate is partially missing following extraction of the tooth.

Type 3: The facial soft tissue and the buccal plate of the bone are both markedly reduced after tooth extraction.

This classification enabled ordering and classifying of post-extraction sockets. A recently published sub-classification of type $2^{(9)}$ now allows even greater clarity to plan regeneration; type 2 presents intact facial soft tissue.

Type $2 \mathrm{~A}$ : Absence of the coronal one-third of labial bone plate of the extraction socket $5 \mathrm{~mm}$ to $6 \mathrm{~mm}$ from the free gingival margin.

Type 2 B: Absence of the middle to coronal two-thirds of the labial bone plate of the extraction socket approximately $7 \mathrm{~mm}$ to $9 \mathrm{~mm}$ from the free gingival margin.

Type $2 \mathrm{C}$ : Absence of the apical one-third of the labial bone plate of the extraction socket $10 \mathrm{~mm}$ or more from the free gingival margin.

The purpose of this study is to show the resolution and control at 21 months of a clinical report where the buccal plate was regenerated using the "ice cream cone" and L-PRF technique in an atraumatic dental extraction followed by immediate placement of a Tapered Internal (Biohorizons, Birmingham, USA) implant in an infected site.

\section{CLINICAL CASE AND TREATMENT}

We present a clinical case of a 53-year-old female patient, Asa II, with osteopenia, in treatment for 5 months with Alendronate Acid $70 \mathrm{mg}$ : one dose weekly. For five years, she had been taking ELCAL-D (500 $\mathrm{mg}$ of Calcium and $800 \mathrm{UI}$ Vitamin, Andromaco Laboratory, Chile), twice a day. She had no history of allergies, smoking or alcohol. The clinical examination of the tooth 2.1 showed an active fistula and mobility of the crown, which presented a metal ceramic crown. The image exam confirmed a vertical fracture treaching to the middle third of the root (Figure 1 - 2).

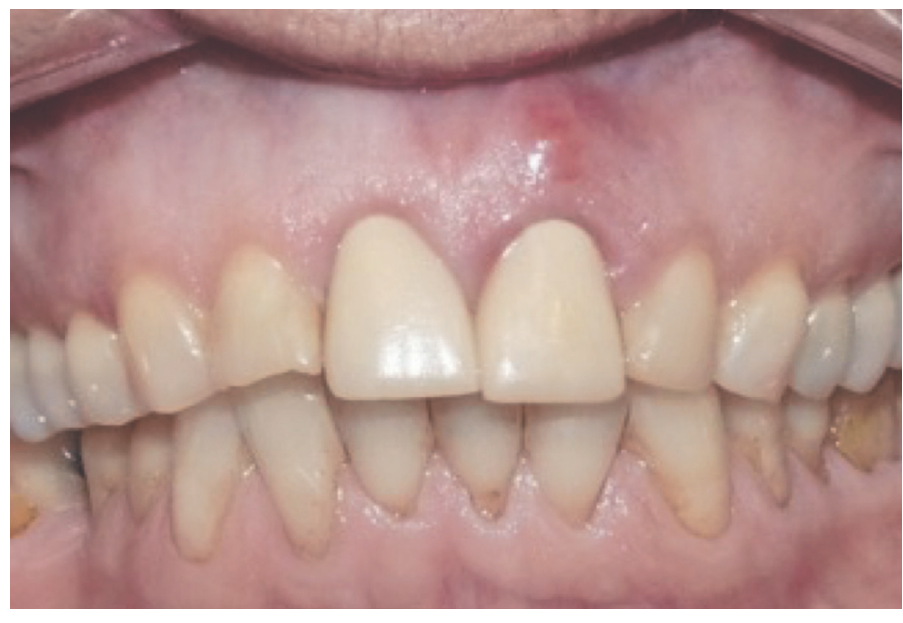

Figure 1. Patient has an active fistula in relation to the tooth 2.1

The patient was premedicated with Amoxicillin 875mg (Optamox, Pharma Investi Laboratory, Chile). She was prescribed one coated tablet every 12 hours for seven days, beginning treatment 24 hours before surgery and taking $125 \mathrm{mg}$ of lysine clonixinate (Nefersil, Pharma Investi Laboratory, Chile) every eight hours for three days 1 hour prior to surgery, 


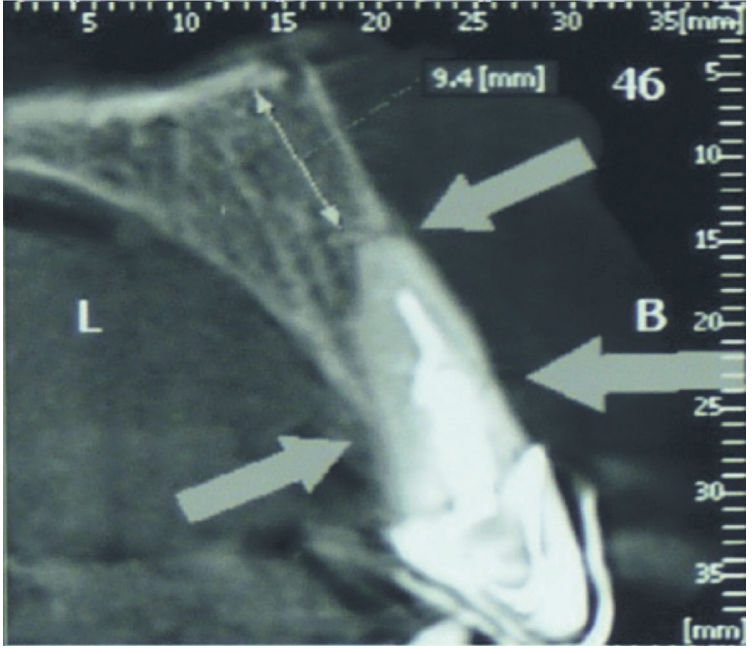

Figure 2. Cone Beam confirms the fracture of the middle third root.

and rinses with chlorhexidine $0.12 \%$ (Perio Aid, Dentaid Laboratory, Spain) every 12 hours for 15 days, beginning 24 hours before surgery.

\section{ATRAUMATIC EXTRACTION AND IMMEDIATE IMPLANT PLACEMENT INTO INFECTED SITE}

The surgery started with an infiltrative technique (Septanest, Articaine 1/100000, Septodont, France). For the extraction of tooth 2.1, thick anterior straight forceps were used. The syndesmotomy was carefully performed with a periotome, and the movement performed with the forceps was rotative to extract the crown and preserve the buccal plate. An atraumatic extractor (Neodent, Curitiba, Brazil) was used to extract the root, obtaining an atraumatic extraction of the fragment, without compromising the plates or the remnant bone (Figure 3 ). The socket was treated by mechanical action and abundant irrigation with physiological saline to remove any inflammatory and infectious tissue that may have remained in the socket.

Clinically, the presence of a buccal defect was verified (Figure 4). However, the gingival architecture was preserved; a Tapered Internal (Biohorizons, Birmingham, USA) $3.8 \mathrm{~mm} \times 15 \mathrm{~mm}$ implant was indicated to develop the "ice cream cone." For that purpose, the implant was placed according to the manufacturer's protocol, using a surgical guide, and left with its closure cap.

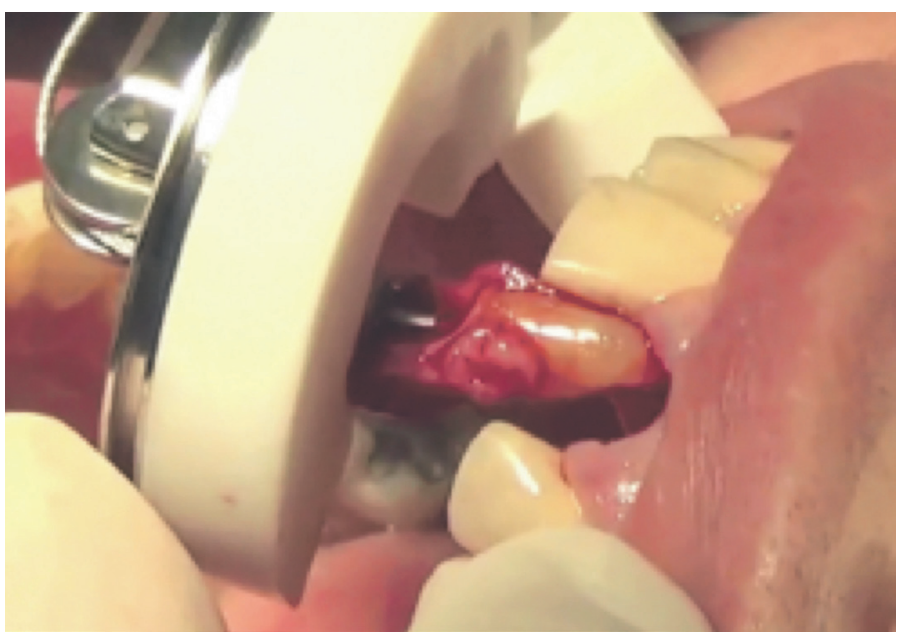

$\overline{\text { Figure 3. The use of an atraumatic extractor (Neodent, Curitiba, Brazil) allowed }}$ the atraumatic removal of the root fragment.

\section{BONE REGENERATION SURGERY “ICE CREAM CONE” AND L-PRF}

Once the implant was placed, a pocket with total thickness was made in relation to the buccal plate. A $15 \mathrm{~mm} \times 20 \mathrm{~mm}$ Mem Lok Biohorizons membrane was then used, which was cut into the shape of "ice cream cone" to regenerate the plate. The membrane was placed over the buccal plate remain, between the periosteum and the remaining bone, using

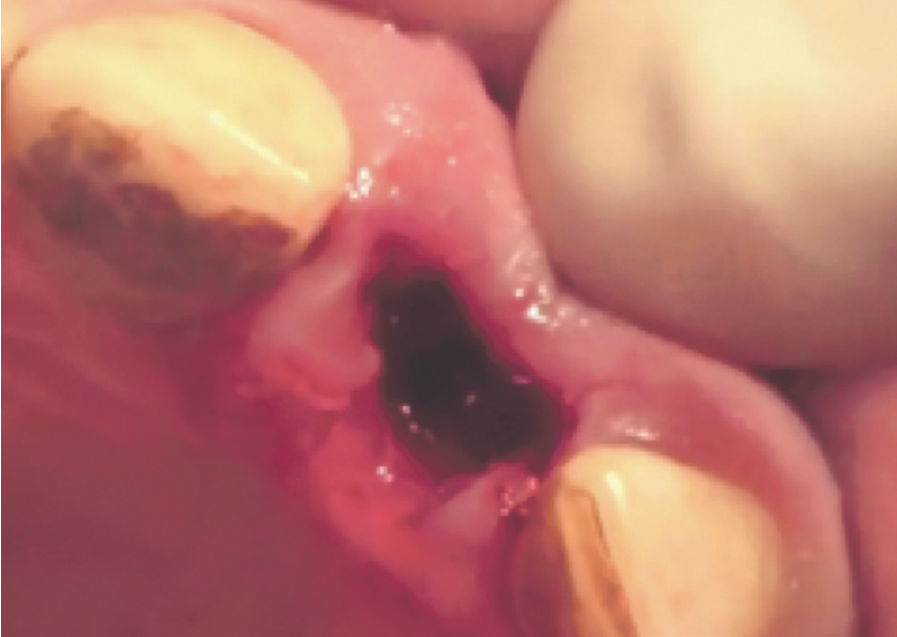

Figure 4. The test checks for buccal plate loss. The socket is Class $2 \mathrm{C}$.

position sutures made with a 5-0 resorbable Polysorb suture, $16 \mathrm{~mm}$ needle, and shear back (Syneture, USA) knotted with a buccal horizontal Mattress. The bone filler used was Mineross Biohorizons, which was placed in the gap between the implant and the buccal contour. The filling material was compacted, taking care not to bend or damage the membrane. After filling the entire socket, the coronal part of the Mem Lok Biohorizons membrane was folded to seal the socket, then sutured with Polysorb 5-0 on the palate using position sutures.

A blood sample was taken prior to surgery, which was then centrifuged according to Choukrun ${ }^{(15)}$ protocols at $2700 \mathrm{RPM} \times 12$ minutes. A fibrin clot was removed to form an L-PRF membrane, which was used to protect the Mem Lok Biohorizons membrane, and to seal the coronal portion of the socket. The L-PRF membrane was installed over the collagen membrane in buccal and palatine with Polysorb 5-0 by position sutures (Figure 5).

Once the surgery was finished, the patient was left with a temporary Maryland bridge to avoid compressing the surgical area.

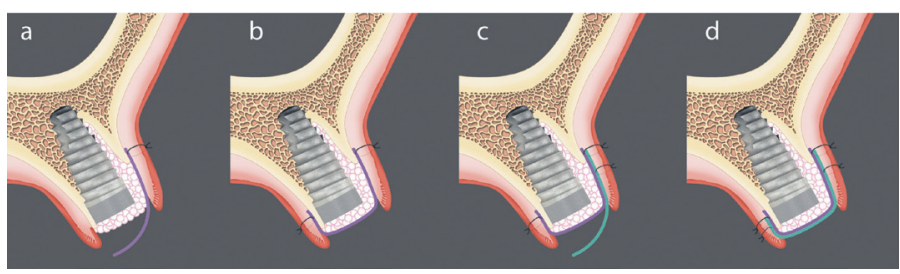

Figure 5. a) The "Ice cream cone" technique is used to regenerate a $2 \mathrm{C}$ defect with the installation of a Biohorizons Tapered Internal $($ implant. b) Mineross $\AA$ and a Memlok® Membrane (lilac) are used, which are placed in a pocket with buccal and palatal position sutures. c) An L-PRF Membrane (green) is positioned inside the pocket above the Memlok® Membrane (lilac) by position sutures. d) Finally, the L-PRF Membrane (green) is suture in Palatine protecting the Memlok® membrane (lilac).

\section{CONNECTION SURGERY AND FINAL REHABILITATION}

The patient was premedicated with lysine clonixinate $125 \mathrm{mg}$ every eight hours for three days (Nefersil, Pharma Investi Laboratory, Chile) and one hour prior to surgery. The area was rinsed with chlorhexidine $0.12 \%$, (Perio Aid, Dentaid Laboratory, Spain) every 12 hours for 15 days, beginning 24 hours before surgery.

Second stage surgery was performed seven months after the implant was installed. To perform the connection surgery, an infiltrative technique (Septanest, Articaine 1/100000, Septodont, France) was used with a $15 \mathrm{c}$ scalpel (Hu-Fridey, USA). A supracrestal linear incision was performed without compromising proximal papillae. A Biohorizons healing abutment was carefully inserted, regular emergency profile and $3 \mathrm{~mm}$ high. The total thickness flap was small and no discharges or sutures were necessary.

The rehabilitation was completed eight months after second-stage surgery. For aesthetic and biomechanical reasons, the tooth 1.1 was rehabilitated with a single-unit metal-ceramic crown. The rehabilitation of the implant 2.1 was developed with Phibo (Barcelona, Spain) system to perform a non-segmented screw metal ceramic crown. Torque of $25 \mathrm{~N}$ was applied, the access of the screw was closed with teflon and composite (Figure 6 - 7). Finally, the patient was left with a restrictive organic occlusal plane. 


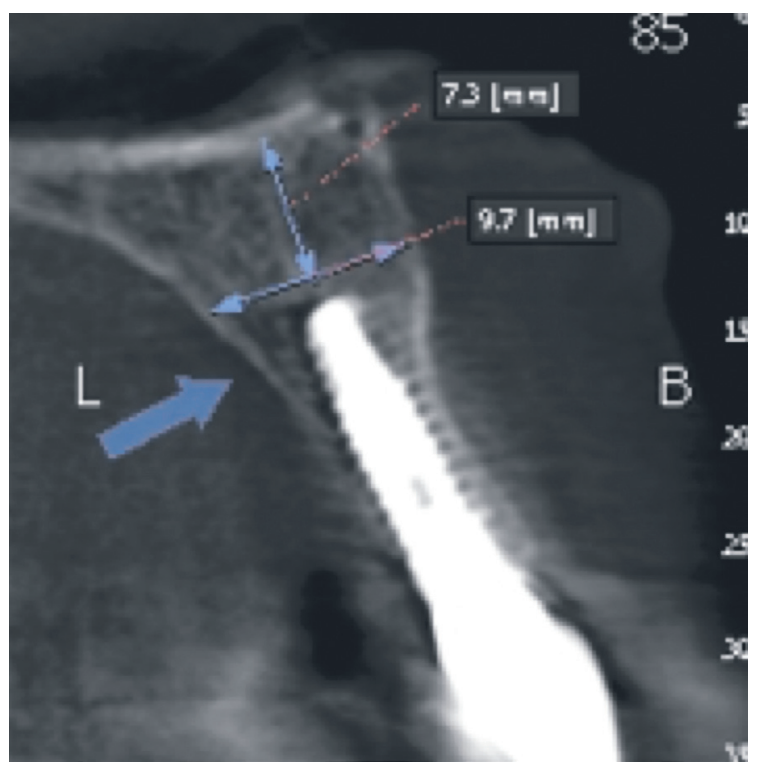

Figure 6. Cone Beam control at 21 months after the implant was installed, which shows the formation of a new buccal plate.

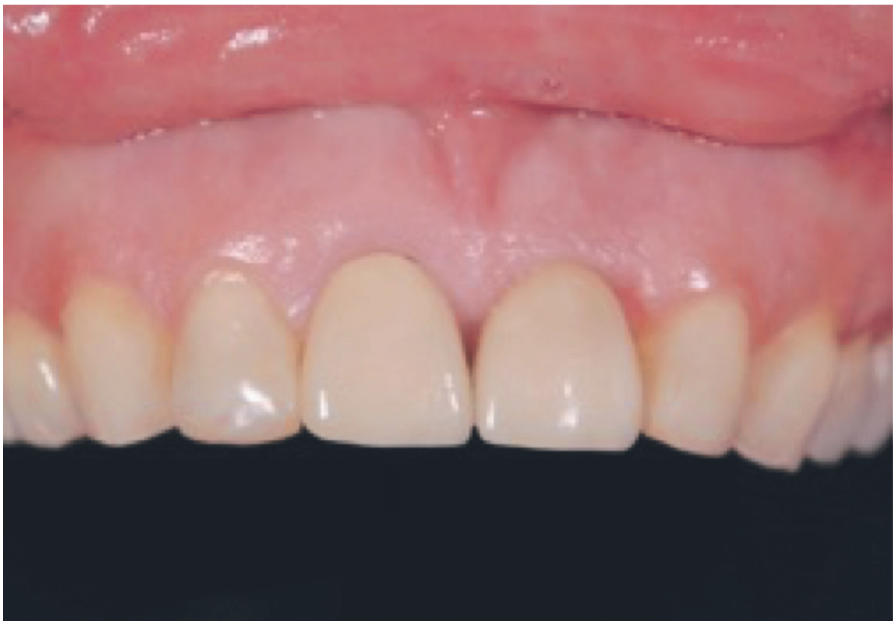

Figure 7. Control at 21 months. The final restoration metal ceramic crown of tooth 1.1 and screwed metal ceramic crown into the implant 2.1 is observed.

\section{DISCUSSION}

At present, an extraction can be considered truly atraumatic only when it leaves intact the surrounding tissues, both the soft and surrounding bone tissue. In this particular clinical case, the combination of an anterio straight forceps, a periotome and an atraumatic extractor (Neodent Curitiba, Brazil) were used. This procedure allowed an easy and vertical extraction, avoiding dilatation of the buccal plate or bone expansion which occurs when a conventional exodontia is performed. The fresh socket is left with an optimal gingival contour. However, various methods have been described in the literature such as endodontic files for root remain, or even the use of piezosurgery ${ }^{(6)}$. In the present case, the root fracture produced a buccal fistula, thus turning the fresh socket into an infected site. It is true the immediate placement of an implant in a fresh socket with sufficient basal bone to be able to anchor an implant is the usual method. This case was special, however; there was a loss of the buccal plate but not of the gingival contour, although we must specify if there was a perforation corresponding to the fistula: According to the classification of Elian ${ }^{(8)}$, the remaining socket corresponded to a class 2 , and particularly $2 \mathrm{C}$, according to the Chu modification ${ }^{(9)}$

In general, several animal and human studies have supported the placement of implants in an infected site, finding no significant differences between implant placement in infected sites and implant placement in non-infected sites ${ }^{(5,6)}$. These studies have been varied, and in general all follow certain guidelines, such as the use of antibiotics, site stripping or mechanical management of the same, and abundant irrigation. With respect to antibiotics, although the dose, type, and time taken are not very clear, the important thing seems to be to indicate them ${ }^{(11)}$. In this study, the patient was premedicated with lysine clonixinate $875 \mathrm{mg}$ (Optamox, Pharma Investi Laboratory, Chile): one cover tablet every 12 hours for seven days, with treatment initiated 24 hours before surgery. Although in this study the irrigation was done with physiological saline in combination with mechanical spooning of the alveolus in alternate forms, other studies have used PRGF(10), chlorhexidine $0.12 \%$, hydrogen peroxide $20 \%$ in combination with laser irradiation $\left(\mathrm{Er}, \mathrm{Cr}\right.$ : YSGG) ${ }^{(5)}$. According to Crispi et al. ${ }^{(12)}$, the high success rate of immediate implants placement in infected sites can be explained by the endoperiodontal origin of the infections, which is associated with anaerobic bacteria commonly restricted to root canal infections, such as Fusobacterium, Prevotella, Porphyromonas, Actinomyces, streptococcus and peptostreptococcus.

In this case, a Tapered Internal (Biohorizons, Birmingham, USA) implant of $3.8 \mathrm{~mm} \times 15 \mathrm{~mm}$ was placement, according to the instructions given by the manufacturer, obtaining an insertion torque of $40 \mathrm{~N}$. According to the aforementioned the alveolus corresponded to a class $2 \mathrm{C}$ reason why a technique of bone regeneration was anticipated and planned in order to recover the buccal plate and thus to maintain the esthetic contour. The "ice cream cone" technique was used, although it was initially designed to regenerate a fresh extraction socket without the immediate implant installation, as mentioned in the publication.7 Another difference from the original technique was that a full thickness pocket was made in both buccal and palatine in order to place the membrane outside the underlying bone tissue by position sutures. In this way, the 15 x $20 \mathrm{~mm}$ Mem lok (Biohorizons, Birmingham, USA) collagen membrane, previously cut with the "ice cream cone" design, was positioned outside the alveolus between the underlying bone and the periosteum, and not inside the alveolus as originally described. The allograft used to close the buccal gap was the Mineross (Biohorizons, Birmingham, USA), which was carefully compacted without bending or retracting the membrane. For this reason, the membrane should be placed first, since the reverse would be very difficult to place the membrane and control the graft material. In order to improve regeneration conditions, the Mineross (Biohorizons, Birmingham, USA) grafting material was hydrated with the exudate liquid from the L-PRF clots obtained by crushing the membranes, according to the protocol developed by Choukrun ${ }^{(15)}$. In addition, an L-PRF membrane was positioned by position sutures inside the pocket created in both the buccal and palatine, over the Mem lok (Biohorizons, Birmingham, USA) membrane in order to protect it mechanically and to take advantage of the reparative potential of L-PRF.

Del Fabro ${ }^{(10)}$ published in 2009 good results in a case of installation of infected implants when using PRGF (plasma rich in growth factors).

The bone resorption of the buccal plate has been well described; it can cause aesthetic defects in the gingival contour ${ }^{(1)}$. Thus, other techniques have also been described for preserving the buccal contour, such as leaving a fragment of the buccal root portion in conjunction with the implant placement, as described by Hürzeler et al.(13) or the threelayered tuberosity grafting technique described by Da Rosa. ${ }^{(14)}$ Finally, opting for the flapless technique of an "ice cream cone" is guaranteed not to mobilize tissue or discharges or cause changes in gingival architecture. The regeneration of a bone contour was controlled with a clinical examination and a cone beam at 21 months after the implant was installed. However, future follow-ups should be performed to control the stability of regenerated buccal tissue with the "ice cream cone technique." Future studies should compare different types of bone filler material, and a greater number of cases should be studied and followed in time.

\section{CONCLUSIONS}

According to the present work, it can be concluded that the regeneration of the buccal plate using the "ice cream cone" technique was possible using Mineross (Biohorizons, Birmingham, USA), Mem Lok (Biohorizons, Birmingham, USA) and L-PRF in the dental extraction and by the immediate installation of a Tapered Internal (Biohorizons, Birmingham, USA) implant in an infected site. In addition, the preservation of the gingival architecture and the maintenance of all these results were sustained 21 months after implant installation. 


\section{References}

1. Araujo M, Lindhe J. Dimensional ridge alterations following tooth extraction. An experimental study in the dog. J Clin Periodontol 2005;32:212-218.

2. Blus C, Szmukler-Moncler S, Khoury P, Orrù G. Immediate implants placed in infected and noninfected sites after a traumatic tooth extraction and placement with ultrasonic bone surgery. Clin Impl Dent Rel Res 2013;17:287-297.

3. Del Fabro M, Ceresoli V, Taschieri S, Ceci C, Testori T. Immediate loading of post extraction implants in the esthetic area: systematic review of the literature. Clin Implant Dent Relat Res. 2015;17:52-70.

4. Noelken R, Neffe BA, Kundel M, Wagner W. Maintenance of marginal bone support and soft tissue esthetic at immediately provisionalised OsseoSpeed implants placed into extraction sites: two-year results. Clin Oral Implants Res 2013;14:214-220.

5. Montoya V, Castillo R, Torres C, Lynch C, Gutiérrez J, Torres D. Outcome of single immediate implants placed in post-extraction infected and non-infected sites, restores with cemented crowns: A three-year prospective study. Journal of Dentistry 2014:42:645-652.

6. Ramos B, Delany M, Wennerberg A. Immediate placement of implants into infected sites: A systematic review. Clin Implant Dent Relat Res 2015;17:1-16.

7. Tan-Chu J, Tuminelli F, Kurtz K, Tarnow D. Analysis of buccolingual dimensional changes of the extraction socket using the "ice cream cone" flapless grafting technique. J Periodontics Restorative Dent 2014;34:399-403.

8. Elian N, Cho S-C. Froum S, Smith R, Tarnow D. A simplified socket classification and repair technique. Pract Proced Aesthet Dent 2007;19:99-104.
9. Chu S, Sarnachiaro G, Hochman M, Tarnow D. Subclassification and clinical management of extraction sockets with labial dentoalveolar dehiscence defects. Compendium 2015;36:516-525.

10. Del Fabbro M, Boggian C, Taschieri S. Immediate implant placement into fresh extraction sites with chronic periapical pathologic features combined with plasma rich in growth factors: preliminary results of single-cohort study. J Oral Maxillofac Surg 2009;67:2476-2484.

11. Corbella S, Taschieri S, Del Fabro M. Postextraction implant in sites with endodontic infection as an alternative to endodontic retreatment: A review of literature. Journal Oral Implantology 2003;39:399-405.

12. Crespi R, Capparè $P$, Gherlone E. Fresh socket implants in periapical infected sites in humans. Journal of Periodontology 2010;81:378-383.

13. Hürzeler M, Zuhr O, Schupbach $P$, Rebele $S$, Emmanouilidis N, Fickl S. The socket shield technique: a proof of principle report. J Clin Periodontol 2010;37:855862 .

14. Da Rosa J, De Oliveira A, Fadanelli M, Sotto-Maior B. Immediate implant placement, reconstruction of compromised sockets, and repair of gingival recession with a triple graft from the maxillary tuberosity; A variation of the immediate dentoalveolar restoration technique. J Prosthet Dent 2014;112:717-722.

15. Choukroun J, Adda F, Schoeffler C, Vervelle A. Une opportunitè en paroimplantologie: le PRF Implantodontie 2000;42:55-62. 